\title{
Avaliação de prescrições medicamentosas de um hospital universitário brasileiro
}

\author{
Evaluation of prescription data of a Brazilian \\ teaching hospital
}

\author{
Joice Mara Cruciol-Souza ${ }^{1}$ \\ João Carlos Thomson ${ }^{1}$ \\ Douglas Godoy Catisti ${ }^{1}$
}

\author{
PALAVRAS-CHAVE \\ - Prescrição de Medicamentos. \\ - Erros de Medicação. \\ - Hospital. \\ - Adultos. \\ - Revisão de Uso de Medicamentos. \\ - Ensino. \\ - Legislação.
}

Recebido em: 30/06/2007 Reencaminhado em: 14/02/2008 Aprovado em: 10/03/2008
REVISTA BRASILEIRA DE EDUCAÇÃO MÉDICA

188 32(2):188-196:2008

\begin{abstract}
R E S U M O
A prescrição éo ponto de partida para a utilização de medicamentos. O objetivo deste trabalho foi identificar a qualidade das informações presentes nas prescrições das enfermarias de um hospital universitário brasileiro. Foram coletadas 1.785 prescrições medicamentosas de pacientes maiores de 12 anos, de ambos os sexos, internados em enfermaria de 1o de janeiro a 30 de abril de 2004. A amostragem foi feita coletando-se todas as prescrições emitidas em um dia de cada semana durante o período de estudo, observando-se o intervalo de seis dias entre cada coleta. Foram avaliados dados relativos à identificação do paciente, do medicamento e do prescritor. Em 230 (12,9\%) prescrições não havia a idade do paciente. Em $224(12 \%)$ prescrições não constava à assinatura do prescritor. Em $16 \%$ dos medicamentos prescritos foi detectada a falta de pelo menos uma informação relativa à posologia (dose, forma farmacêutica, via e/ou intervalo entre doses). A falta destas informações éum obstáculo ao uso seguro de medicamentos, podendo levar ao uso inadequado e a reações adversas. Sugerimos intervenções educativas junto aos profissionais que lidam com o medicamento (desde a prescrição até sua utilização) e a implantação de monitoramento farmacoterapêutico dos pacientes. A participação mais ativa do farmacêutico na equipe de saúde também pode proporcionar tratamentos mais efetivos, seguros e convenientes aos pacientes hospitalizados. A assistência farmacêutica e a utilização de medicamentos em hospitais brasileiros precisam ser mais estudadas.
\end{abstract}

\section{A B S T R A C T}

Prescription is the starting point for medicine use. The objective of this work was to identify the quality of the information contained in the prescriptions of a Brazilian university hospital. During the period January 1 to April 30, 2004, 1.785 drug prescriptions for inpatients older than 12 years were collected. The sample consisted of the total of prescriptions emitted on one day of each week during the study period, observing an interval of 6 days between each collection. Characteristics of the patient, the drug and the prescriber were evaluated. In 230 (12.9\%) prescriptions the age of the patient was no mentioned. In $224(12 \%)$ cases the prescriber had not signed the prescription. In $16 \%$ of prescriptions at least one item of the information regarding dosage (dosage, pharmaceutical form, route and/or time interval between doses) was missing. The lack of such information represents an obstacle for safe use of medicines and can lead to misuse and adverse reactions. We suggest educative interventions for the professionals who deal with medicines and pharmacotherapeutic monitoring of patients. The participation of the pharmacist in the health team can also contribute to more effective, safe and convenient therapies for the hospitalized patients. Pharmaceutical care and medicine use in Brazilian hospitals need further studies.

\footnotetext{
${ }^{1}$ Universidade Estadual de Londrina, Paraná, Brasil.
} 


\section{INTRODUÇÃO}

A preocupação com problemas de saúde relacionados a medicamentos tem aumentado entre os profissionais da saúde ${ }^{1}$. No Brasil, medidas como a Política Nacional de Medicamentos², os medicamentos genéricos ${ }^{3}$, o controle de propagandas ${ }^{4}$ e outras medidas da Agência Nacional de Vigilância Sanitária (Anvisa) são exemplos da valorização deste tema.

Kohn e colaboradores ${ }^{5}$ estimaram a ocorrência de muitas mortes anualmente devidas a erros relacionados com o uso inadequado de medicamentos. Revisões sistemáticas apontaram que $5 \%$ dos pacientes admitidos para internamento hospitalar são atingidos por erros relacionados com o uso de medicamentos $^{6,7}$. Segundo a United States Pharmacopeia Convention ${ }^{8}$, mais de $10 \%$ dos erros com medicamentos no ano 2000 ocorreram por falhas na documentação e por defeitos na comunicação interpessoal entre os profissionais da saúde e os pacientes. Apesar da importância deste tema, no Brasil, ainda são poucos os estudos que o exploram.

A dispensação de medicamentos é o ato farmacêutico de prover um ou mais medicamentos a partir de uma receita ${ }^{2}$. Durante este ato, o farmacêutico deve avaliar a prescrição sob os seguintes aspectos: apresentação e qualidade da informação, cumprimento da legislação e qualidade terapêutica, ${ }^{9,12}$.

O objetivo deste trabalho foi avaliar prescrições da enfermaria de um hospital universitário brasileiro com relação à apresentação da informação sobre o paciente, o prescritor e os medicamentos, bem como o cumprimento da legislação vigente. Este trabalho pretende contribuir com dados farmacoepidemiológicos para o crescimento da assistência farmacêutica em nosso país e valorizar a importância da prescrição médica.

\section{MATERIAL E METODOLOGIA}

\section{Desenho do estudo}

Este foi um estudo transversal de utilização de medicamentos com base na prescrição medicamentosa. Foi realizado com os dados das prescrições dispensadas pela Farmácia Hospitalar do Hospital Universitário da Universidade Estadual de Londrina. O hospital em questão possui 300 leitos, sendo cem os leitos de enfermaria destinados a pacientes maiores de 12 anos. Este hospital está ligado a uma instituição pública de ensino e é também centro de referência para cuidados médicos hospitalares na região norte do estado do Paraná.

\section{População em estudo}

Durante quatro meses, cerca de 11.250 pacientes adultos internados na enfermaria do hospital receberam prescrições ma- nuscritas que continham dois ou mais medicamentos. O total de prescrições estudado foi de 1.785, e o número total de medicamentos prescritos foi de 13.056.

\section{Critério de inclusão}

Prescrições de enfermaria com dois ou mais medicamentos foram selecionadas uma vez por semana, de janeiro a abril de 2004. Todos os grupos de medicamentos foram aceitos. Pacientes de ambos os sexos e com idade acima de 12 anos foram incluídos no estudo. Foi incluída apenas uma prescrição de cada paciente durante sua hospitalização no período de estudo.

\section{Protocolo de estudo}

No hospital em estudo, as prescrições são manuscritas em duas vias: a prescrição original (que permanece no prontuário do paciente) e uma cópia carbonada (que segue para a farmácia hospitalar). O sistema de distribuição de medicamentos no hospital é do tipo "individual de 24 horas", com exceção dos quimioterápicos antineoplásicos, que seguem o tipo "dose unitária".

As prescrições foram selecionadas a partir de sua cópia carbonada para as análises. A amostragem foi feita coletando-se todas as prescrições emitidas em um dia de cada semana durante o período de estudo, com intervalo de seis dias entre cada coleta. A razão paciente masculino/feminino no estudo foi preservada em 60/40, de acordo com o número de leitos das respectivas enfermarias do hospital. Todos os medicamentos foram classificados pela Anatomical-Therapeutic-Chemical Classification (ATC), disponibilizada e atualizada anualmente pela Organização Mundial da Saúde ${ }^{13}$.

\section{Método para avaliação das prescrições}

A Figura 1 apresenta o fluxograma do tipo top-down ${ }^{14}$, utilizado como guia para a avaliação da prescrição medicamentosa nos quesitos apresentação da informação e cumprimento da legislação.

a) Características do paciente: sexo, idade, presença ou não do número de registro do paciente no hospital, presença ou não da identificação do quarto e do leito ocupado pelo paciente.

b) Características do prescritor: clínica responsável pelo internamento do paciente, número de prescritores em cada prescrição, presença ou não da assinatura.

c) Características dos medicamentos prescritos: quantidade de medicamentos por prescrição, identificação pelo nome genérico, presença ou não da dose, presença ou não da forma farmacêutica, presença ou não da via de administração, presença ou não do intervalo entre doses. 
FIGURA 1

Fluxograma do tipo top-down para avaliação de prescrição medicamentosa ${ }^{4}$.

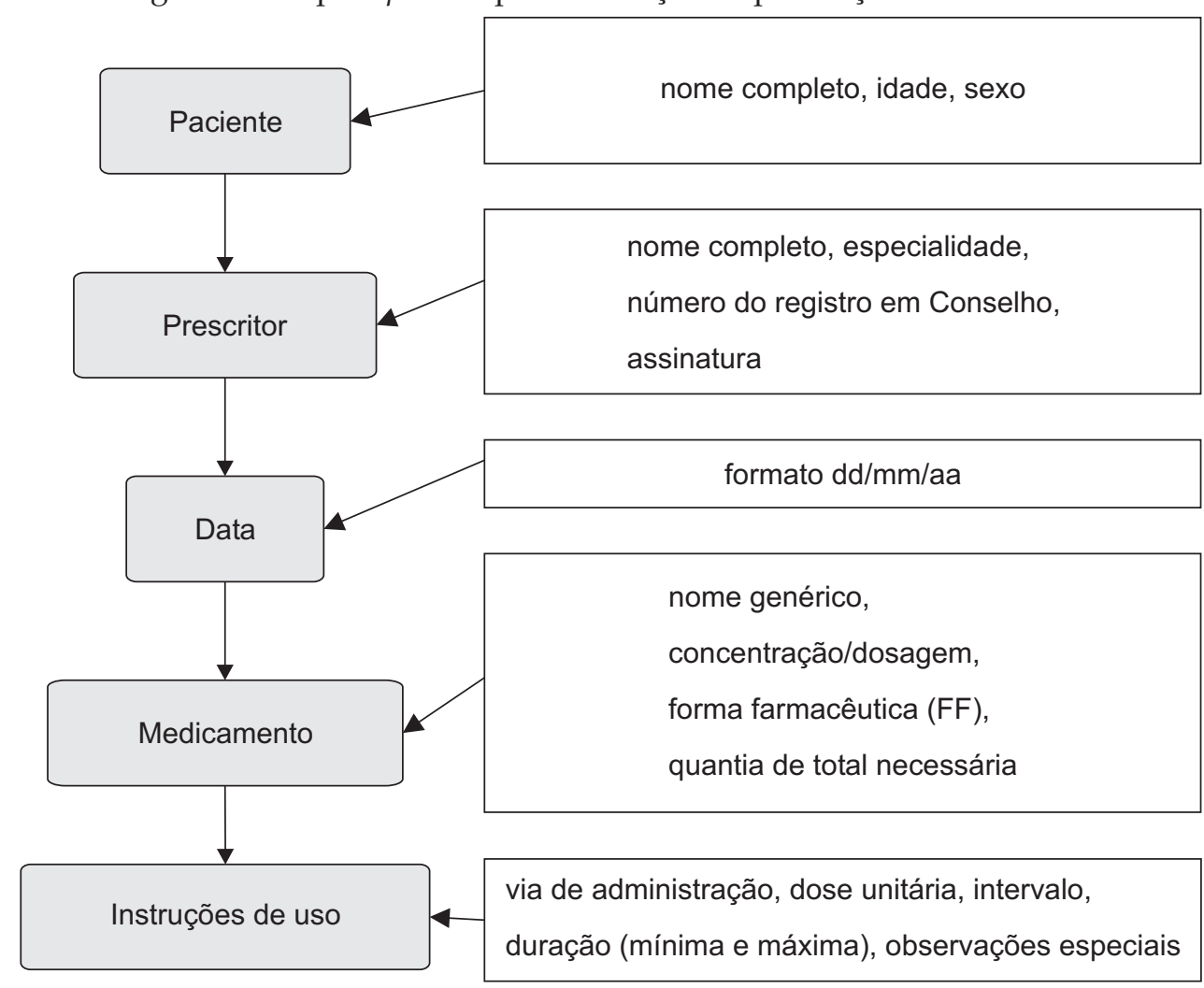

\section{Análise estatística}

Dados demográficos dos pacientes e dados quantitativos da prescrição foram apresentados como média, desvio padrão e porcentagem. Os dados foram digitados em planilhas Excel『 versão 7.0, onde foram realizados todos os cálculos apresentados.

\section{Comitê de ética}

O trabalho foi previamente aprovado pelo comitê de ética local e fez parte de um estudo farmacoepidemiológico maior, envolvendo interações medicamentosas ${ }^{15}$.

\section{RESULTADOS}

Do total de 1.785 prescrições avaliadas, 1.089 (61,0\%) prescrições foram destinadas a pacientes internados na unidade masculina e 696 (39,0\%) na feminina. A idade dos pacientes variou de 12 a 98 anos, com média de $52,7 \pm 18,9$ anos e mediana de 54 anos. A faixa etária dos pacientes mais freqüente na amostra de prescrições da enfermaria foi a de 50 a 59 anos em ambos os sexos. A idade do paciente foi uma informação que estava presente em 1.555 prescrições $(87,1 \%)$.
Nos dados referentes aos pacientes, encontramos que em 230 prescrições (12,9\%) a idade do paciente não estava informada; em 96 (5,4\%) não havia o número de registro do paciente no hospital; e em $6(0,3 \%)$ não havia a informação quanto ao quarto e leito ocupados pelo paciente.

A clínica responsável pelo internamento do paciente foi identificada em 1.757 prescrições $(98,4 \%)$ da amostra estudada. As clínicas mais freqüentes foram: Ortopedia (269; 15,1\%); Clínica Geral (176; 9,9\%); Cirurgia do Aparelho Digestivo (170; 9,5\%); Pronto Socorro Cirúrgico (146; 8,2\%); Neurologia Clínica (143; 8,0\%); e Cardiologia (141; 7,9\%).

A assinatura do prescritor esteve presente em 1.571 prescrições $(88,0 \%)$. Em um terço do total das prescrições $(615 ; 34,5 \%)$, havia a assinatura de mais de um prescritor, chegando a até quatro. Entretanto, em 214 prescrições (12,0\%) não havia a assinatura de qualquer prescritor.

As prescrições apresentaram a variação de 2 a 26 medicamentos prescritos. Em média, cada prescrição apresentou $7 \pm 4$ medicamentos, ficando a mediana também em sete medicamentos. $\mathrm{O}$ total de prescrições examinadas (1.785) apresentou 13.056 medicamentos prescritos. Dos 13.056 medicamentos da amostra, 
$6.291(48,2 \%)$ foram prescritos pelo nome genérico. Os dez medicamentos prescritos com maior freqüência foram: dipirona, metoclopramida, omeprazol, tramadol, insulina, ranitidina, captopril, enoxaparina, heparina e ácido acetilsalicílico.

As informações de 2.089 (16\%) medicamentos prescritos não estavam completas. A dose que deveria ser administrada não foi indicada em 148 medicamentos (1,1\% de 13.056). A forma farmacêutica que deveria ser utilizada para administração do medicamento ao paciente foi identificada em 11.741 medicamentos prescritos $(88,9 \%)$. Ou seja, esta informação esteve ausente em 1.315 medicamentos (10,1\% de 13.056). A via de administração não esteve indicada para 392 (3,0\%) medicamentos prescritos. As três vias mais prescritas foram: oral $(46,9 \%)$, intravenosa $(35,2 \%)$ e subcutânea $(8,5 \%)$. Por fim, os intervalos entre doses para utilização dos medicamentos não foram definidos na prescrição para $261(2,0 \%)$ medicamentos.

As Tabelas 1 e 2 resumem os resultados apresentados neste trabalho.

TABELA 1

Informações ausentes referentes ao paciente e ao prescritor detectadas em 1.785 prescrições medicamentosas do Hospital Universitário da Universidade Estadual de Londrina

\begin{tabular}{lcc}
\hline Categoria da Informação & Informação Ausente & $\mathrm{N}(\%)$ \\
\hline \multirow{3}{*}{ Paciente } & Idade & $230(12,8)$ \\
& Registro interno & $96(5,4)$ \\
Prescritor & Quarto e leito & $6(0,3)$ \\
& Clínica de internamento & $28(1,6 \%)$ \\
\hline
\end{tabular}

\section{TABELA 2}

Informações ausentes referentes ao total de 13.056 medicamentos presentes em prescrições medicamentosas do Hospital Universitário da Universidade Estadual de Londrina

\begin{tabular}{ccc}
\hline Categoria da Informação & Informação Ausente & N $(\%)$ \\
\hline \multirow{2}{*}{ Medicamentos } & Nome genérico & $6765(51,8)$ \\
& Dosagem & $148(1,1)$ \\
& Forma farmacêutica & $1315(10,1)$ \\
& Via de administração & $392(3,0)$ \\
& Intervalo entre doses & $261(2,0)$ \\
\hline
\end{tabular}




\section{DISCUSSÃO}

O uso inadequado de medicamentos é freqüente e sério, sendo responsável, nos Estados Unidos, por mais de 218 mil mortes, 9 milhões de hospitalizações e gastos em torno de US\$ 177 bilhões anuais ${ }^{16,17}$. Segundo a metanálise de Lazarou e colaboradores $^{18}$, as reações adversas aos medicamentos estiveram entre as três primeiras causas de morte nos Estados Unidos.

A qualidade da informação encontrada, com ausência de alguns dados, pode acarretar problemas relacionados com medicamentos, de complexidade e magnitude diferentes. A qualidade da assistência farmacêutica e a documentação legal dos pacientes também ficam prejudicadas. As conseqüências podem variar desde o aumento no tempo de permanência hospitalar até a elevação das taxas de mortalidade ${ }^{1,7}$.

A prescrição exerce papel fundamental no tratamento medicamentoso. Um primeiro fator que lhe confere tanta importância, principalmente no ambiente hospitalar, é a interligação que ela exerce entre toda a equipe de saúde. Outro fator, intimamente relacionado ao primeiro e até mais importante que aquele, reside em que a prescrição é o instrumento que garante a execução dos pilares do uso correto de medicamentos: paciente certo, medicamento correto, na quantidade certa e na hora certa ${ }^{19}$.

A dispensação de medicamentos permite ao farmacêutico avaliar a receita médica sob diferentes aspectos ${ }^{14}$. Em alguns casos, a ausência de informações sobre a forma farmacêutica ou dosagem pode não ser essencial. Isto ocorre quando há apenas um produto disponível na padronização do hospital com aquele fármaco prescrito. Nestes casos, embora a prescrição esteja incompleta, o paciente não chega a sofrer as conseqüências desta falta de informação. Entretanto, muitas vezes tem-se mais de um produto padronizado no hospital para determinado fármaco, com formas farmacêuticas e dosagens diferentes, para administração tanto oral quanto parenteral. Assim, é essencial a identificação do prescritor para sanar tais dúvidas.

A presença de dados de identificação do paciente, do prescritor e dos medicamentos, a legibilidade da receita e o cumprimento da legislação acabam por influenciar a qualidade terapêutica.

Durante a dispensação, o farmacêutico pode dar ao usuário do medicamento ou à equipe médica elementos importantes, como a ênfase no cumprimento do regime terapêutico e cuidados com as interações medicamentosas e reações adversas ${ }^{20,23}$.

No ambiente hospitalar, muitas vezes, a etapa de orientação sobre os medicamentos acaba sendo omitida, e grande parte das dúvidas a respeito de uma prescrição médica incompleta ou mal grafada necessita ser discutida com o próprio prescritor. Cabe a ele tomar as decisões referentes àquilo que foi prescrito, valorizando esta atribuição tão própria do exercício da medicina. Assim, torna-se evidente que a correta identificação do prescritor é uma medida que visa agregar valor à prescrição e à função do médico na equipe hospitalar. A assinatura do prescritor é também uma exigência legal na documentação do paciente ${ }^{9,24}$. Saber a especialidade médica, a partir da clínica responsável pelo internamento do paciente, pode ser uma ferramenta útil para se chegar ao prescritor, porém esta informação também esteve ausente em $1,6 \%$ das prescrições. A falta de assinatura na prescrição, bem como da clínica de entrada do paciente, pode ferir todo o princípio de Galeno, já que as dúvidas sobre qualquer ponto da prescrição não conseguirão ser sanadas pelo profissional responsável.

A falta de informação na prescrição pode interferir com a comunicação interprofissional, prejudicando-a, e pode levar a erros de medicação ao paciente ${ }^{25}$. Prescrições incompletas impedem a eficiência do trabalho de dispensação dos medicamentos, colocando em risco a qualidade da assistência farmacêutica ao paciente. Além disso, as falhas de comunicação entre os profissionais detectadas por meio de informações incompletas na prescrição podem ter influenciado negativamente a qualidade dos tratamentos farmacoterapêuticos realizados para estes pacientes no hospital onde o estudo foi realizado.

Alertamos para o fato de que estes dados foram coletados num hospital-escola, onde temos profissionais altamente capacitados no ensino na área da saúde. Como estaria esta informação na rede privada, onde não podemos determinar essa qualificação profissional? Independentemente desta questão, não pretendemos apontar culpados pela qualidade do processo, mas, sim, alertar para que se adotem medidas e condutas de ensino para o uso seguro de medicamentos em todos os locais de trabalho. Seja nos cursos de Medicina, Farmácia ou Enfermagem, acreditamos ser necessário que o ensino sobre medicamentos objetive o uso correto e melhor qualidade nos tratamentos medicamentosos em termos de efetividade, segurança e conveniência.

A ausência de informações sobre o paciente pode fazer com que se administre o medicamento a pessoas erradas, levar à demora na administração do medicamento ao paciente certo e impedir o cálculo dos gastos de cada paciente com medicamentos durante seu internamento. Sebastião ${ }^{26}$ observou dados semelhantes em prescrições hospitalares, porém com índices diferentes dos nossos. Enquanto verificamos a falta de identificação do paciente como um problema mais freqüente, aquele autor 
observou, em maior proporção, a identificação dos prescritores e da clínica de entrada.

"O medicamento... para a pessoa certa"19. Segundo a The United States Pharmacopeial Convention ${ }^{8}$, a administração do medicamento ao paciente errado tem sido a causa de $5 \%$ dos erros relatados com medicamentos. A falta do número de registro do paciente no hospital e/ou do quarto e leito ocupados por ele pode contribuir para a ocorrência deste erro no ambiente hospitalar. A falta de informação quanto à idade do paciente pode impedir a dupla conferência dos dados, que é uma forma de evitar erros $^{27}$. Quando o farmacêutico possui dados completos (como idade, peso e doenças do paciente), pode auxiliar a equipe médica, por exemplo, checando o cálculo das doses diárias prescritas, de modo a otimizar as terapêuticas.

"O medicamento... na quantidade certa"19. Segundo aquele órgão americano ${ }^{8}$, a administração de uma dose inadequada pode corresponder a $23 \%$ dos erros de medicação. A má grafia da vírgula e as transformações de miligramas para gramas ou microgramas, dependendo do caso, podem tornar a dose dez vezes maior ou menor do que a necessária, causando reações adversas ou não alcançando os objetivos farmacoterapêuticos desejados.

"O medicamento certo". Segundo a Anvisa, medicamento é todo "produto farmacêutico, tecnicamente obtido ou elaborado, com finalidade profilática, curativa, paliativa ou para fins de diagnóstico. É uma forma farmacêutica terminada que contém o fármaco, geralmente em associação com adjuvantes farmacotécnicos" 28,29 . Ou seja, quando falamos em "medicamento certo", nos referimos ao fármaco apresentado em determinada forma farmacêutica, para utilização por uma via específica. Este ponto do princípio de Galeno que estamos utilizando como norteador nesta discussão não pode ser contemplado quando é impossível identificar a forma farmacêutica ou a via de administração a ser utilizada para um medicamento. Assim, os pacientes podem receber um medicamento numa forma farmacêutica ou por uma via que não seja a mais adequada a seu caso. O problema poderá se tornar ainda mais crítico se, além deste dado, não pudermos identificar o prescritor.

"Na hora correta..." A adoção de esquemas terapêuticos tidos como "clássicos" ou "usuais" tem muitos aspectos positivos para o funcionamento de um hospital. Entretanto, a ausência de convenções internas pode conduzir a conceitos como o de que "o esquema terapêutico usual é a única forma correta" ou que "não há risco para o paciente quando se utiliza o esquema mais comum", o que pode não ser verdade, pois as reações adversas ocorrem nas doses usuais ${ }^{30}$. A especificação do esquema de ho- rários para a administração da medicação de qualquer paciente deve ser feita de forma individualizada e sempre deve estar presente na prescrição ${ }^{12}$.

\section{ESTRATÉGIAS}

Acreditamos que a integração entre o ensino médico e o farmacêutico deva ser uma meta a alcançar no Brasil. Alguns cursos de Farmácia realizam internatos integrados com cursos de Medicina nos Estados Unidos e Europa, haja vista as grades curriculares destes cursos, disponíveis para consulta na internet ${ }^{31,33}$. Segundo as ementas e programas destes internatos, as equipes multiprofissionais discutem as disponibilidades de tratamento sob as óticas da medicina e da tecnologia farmacêutica para os casos estudados, propiciando análises de risco-benefício e custo-benefício de impacto importante sobre a saúde do paciente.

No Brasil, talvez pelo fato de a legislação proibir a sociedade comercial entre médicos e farmacêuticos ${ }^{34}$, eles acabam por interagir pouco também profissional e academicamente. São raros os hospitais que mantêm farmacêuticos como parte de seu corpo clínico, desenvolvendo algum tipo de monitoramento farmacoterapêutico ou atuando em parceria com as equipes médicas ${ }^{35}$.

Assim, o médico precisa estar ciente de como os efeitos terapêuticos desejados podem estar sendo desviados de suas metas pelos próprios medicamentos. O grande volume de prescrições dispensadas diariamente nos hospitais brasileiros, o reduzido número de farmacêuticos hospitalares, a falta de serviços de informação sobre medicamentos ${ }^{35}$, além do tempo necessário ${ }^{36} \mathrm{e}$ da complexidade de uma avaliação farmacoterapêutica completa do paciente ${ }^{37}$ e dos custos para treinamento de recursos humanos são questões a considerar quando nos referimos a problemas de prescrição medicamentosa no Brasil.

O desenvolvimento de protocolos e rotinas para a prescrição é uma alternativa útil para diminuir as falhas por falta de informações na prescrição ${ }^{38,39}$. O The Institute for Safe Medication Practices ${ }^{40}$ prega a abolição das prescrições manuscritas como uma medida fundamental para maior segurança na utilização de medicamentos. Além da melhora da legibilidade nas prescrições feitas em computador, os softwares desenvolvidos para prescrição computadorizada geralmente permitem a emissão de prescrição totalmente completa, evitando falhas por falta de informação, duplicidades ou interações medicamentosas graves. O desenvolvimento de softwares para prescrição hospitalar adaptados à realidade brasileira é uma alternativa ainda difícil de ser implantada, talvez pelo custo operacional e características da prática em nossa realidade. 
Para o Food and Drug Administration ${ }^{41}$, os erros de prescrição podem ser uma fonte significante de morbidade e mortalidade institucional, sendo importante monitorar a medicação desde seu ponto de origem (a prescrição), para prevenir a ocorrência de incidentes semelhantes.

Erros de medicação podem ser cometidos por qualquer pessoa que lide com medicamentos, e suas causas são variadas. Eis algumas delas: sistema gerencial e de garantia de qualidade ${ }^{42}$; nomenclatura do medicamento ${ }^{43}$; caligrafia médica ${ }^{44}$; distração momentânea; estresse profissional ${ }^{45}$; sistemas de prescrição, transcrição, dispensação e administração de medicamentos; falta de conhecimento ou treinamento inadequado ${ }^{46}$; fontes inadequadas de informação sobre medicamentos ${ }^{47}$; erros humanos ${ }^{5}$. Segundo o Institute of Health Improvement ${ }^{39}$, a prevenção de erros de medicação se dá pelo esforço conjunto multiprofissional na avaliação do uso dos medicamentos e no desenvolvimento de intervenções efetivas.

\section{CONCLUSÃO}

Com este estudo foi possível detectar um número considerável de informações ausentes nas prescrições medicamentosas de um hospital universitário brasileiro, tanto nos dados referentes ao paciente, quanto nos que dizem respeito ao prescritor e aos medicamentos propriamente ditos.

É necessário que a prescrição seja vista como um documento terapêutico de comunicação entre profissionais. Além disso, acreditamos que a valorização da prescrição por meio da melhor utilização deste instrumento pela equipe hospitalar contribuirá para melhor utilização dos recursos financeiros da instituição. O uso correto de medicamentos e a supervisão deste uso podem alterar o tempo de permanência do paciente no hospital e diminuir tratamentos desnecessários de reações adversas que podem ser evitadas ${ }^{1,7}$.

Os dados aqui apresentados de um hospital universitário de referência no Paraná aponta um problema que pode estar presente em muitos outros hospitais brasileiros: a baixa qualidade da prescrição medicamentosa. As equipes de saúde locais devem identificar e discutir as melhores alternativas para eliminar este fator de risco para a saúde dos pacientes internados. Acreditamos, ainda, que a inserção do farmacêutico em discussões clínicas pode contribuir para melhorar a qualidade da prescrição, diminuindo a distância entre o clínico e o farmacêutico, e aumentando a comunicação e integração profissional, respeitando as especialidades de cada um. Assim, o medicamento poderá cumprir fidedignamente sua função no tratamento farmacológico instituído para cada paciente, com eficácia, segurança e conveniência.

\section{REFERÊNCIAS}

1. Hepler CD, Segal R. Preventing medication errors and improving drug therapy outcomes: A management systems approach. Boca Raton (FL): CRC Press; 2003.

2. BRASIL. Ministério de Estado da Saúde. Política nacional de medicamentos. Portaria no 3916 de 30 de outubro de 1998. Aprova a Política Nacional de Medicamentos, cuja íntegra consta do anexo desta Portaria. D.O.U. - Diário Oficial da [República Federativa do Brasil]. Brasília, 10 nov. 1998. [capturado 20 fev. 2007]. Disponível em: http:/ / www.anvisa.gov.br/e-legis /

3. BRASIL. Lei no 9.787, de 10 de fevereiro de 1999. Altera a Lei no 6.360 , de 23 de setembro de 1976, que dispõe sobre a vigilância sanitária estabelece o medicamento genérico, dispõe sobre a utilização de nomes genéricos em produtos farmacêuticos e dá outras providências. Diário Oficial da [República Federativa do Brasil]. Brasília, 11 fev. 1999. [capturado 20 fev. 2007]. Disponível em: http:/ /e-legis.anvisa.gov.br/leisref/public/showAct.php?id=16622\&word =

4. BRASIL. Agência Nacional de Vigilância Sanitária. Resolução RDC nํ102, de 30 de novembro de 2000. Aprova o Regulamento sobre propagandas, mensagens publicitárias e promocionais e outras práticas cujo objeto seja a divulgação, promoção ou comercialização de medicamentos de produção nacional ou importados, quaisquer que sejam as formas e meios de sua veiculação, incluindo as transmitidas no decorrer da programação normal das emissoras de rádio e televisão. Diário Oficial da [República Federativa do Brasil]. Brasília, 01 dez. 2000. [capturado 20 fev. 2007]. Disponível em: http://e-legis.anvisa.gov.br/leisref/public/showAct.php?id=16627\&word=

5. Kohn LT, Corrigan JM, Donaldson MS, editores. To Err is Human: Building a Safer Health System. Washington (DC): Subcommitte on Quality of Health Care in America, Institute of Medicine, National Academy Press; 1999.

6. Bond CA, Raehl CL, Franke T. Medication errors in the Unided States Hospitals. Pharmacotherapy. 2001; 9:1023-36.

7. Wiffen P, Gill M, Edwards J, Moore A. Adverse Drug Reactions in Hospital Patients: a systematic Review of the Prospective and Retrospective Studies. Bandolier Extra. [online] 2002; June. [capturado 19 maio 2005]. Disponível em: http:/ / www.jr2.ox.ac.uk/bandolier/Extraforbando/ADRPM.pdf.

8. U S Pharmacopeial. The Unided States Pharmacopeial Convention. Sumary of information submitted to MedMARX in the year 2000: Charting a course for change. 
Rockville, MD: USP Center for the Advancement of Patient Safety. [capturado 22 out. 2004]. Disponível em: http://www.usp.org/products/medMarx/

9. BRASIL. Ministério da Saúde. Secretaria de Vigilância Sanitária. Portaria n⿳o 344, de 12 de maio de 1998. Aprova o regulamento técnico sobre substâncias e medicamentos sujeitos a controle especial. Diário Oficial da [República Federativa do Brasil]. Brasília, 15 maio 1998. [capturado 20 fev. 2007]. Disponível em: http://e-legis.anvisa.gov.br/leisref/public/showAct.php?id=20054\&word=

10. Brasil. Agência Nacional de Vigilância Sanitária. Resolução nº 328, de 22 de julho de 1999. Dispõe sobre requisitos exigidos para a dispensação de produtos de interesse à saúde em farmácias e drogarias. Diário Oficial da [República Federativa do Brasil]. Brasília, 26 jul. 1999. [capturado 20 fev. 2007]. Disponível em: http://e-legis.anvisa.gov.br/leisref/public/showAct.php?id=1249\&word=

11. BRASIL. Conselho Federal de Farmácia. Resolução n⿳ำ 357, de 20 de abril de 2001. Aprova o regulamento técnico das Boas Práticas de Farmácia. Diário Oficial da [República Federativa do Brasil]. Brasília, 27 abr. 2001. [capturado 20 fev. 2007]. Disponível em: http://e-legis.anvisa.gov.br/leisref/public/showAct.php?id=4123\&word=

12. BRASIL. Ministério de Estado da Saúde. Portaria $n^{\circ} 675$, de 30 de março de 2006. Aprova Carta dos Direitos dos Usuários da Saúde, que consolida os direitos e deveres do exercício da cidadania na saúde em todo o País. D.O.U. - Diário Oficial da [República Federativa do Brasil]. Brasília, 31 mar. 2006. [capturado 20 fev. 2007]. Disponível em: http://e-legis.anvisa.gov.br/leisref/public/showAct.php?id=21900\&word=

13. WHO Collaborating Centre for Drug Statistics Methodology. Anatomical Therapeutic Chemical (ATC) classification [base de dados na internet] Oslo (Noruega): Norwegian Institute of Public Health. c 1982 [capturado 22 out. 2004]. Disponível em: http: / www.whocc.no/atcddd

14. Cruciol-Souza JM. Ensinando com Fluxogramas de Avaliação de Prescrições e o Jogo: Praticando a Dispensação de Medicamentos. Caderno de Resumos do 4. Congresso de Farmácia e Análises Clínicas de Ponta Grossa; 2002 jul. 26-28; Paraná, Brasil. Ponta Grossa, PR: Kugler Artes Gráficas; 2002. p. 29.

15. Cruciol-Souza JM. Prevalência de interações medicamentosas e fatores associados em prescrições do Hospital Universitário da Universidade Estadual de Londrina, no período de janeiro a abril de 2004. Londrina; 2006. Doutorado [Tese] - Universidade Estadual de Londrina.
16. Johnson J, Bootman J. Drug-related morbidity and mortality: A cost-of-illness model. Arch. Int. Med. 1995; 155:1949-56.

17. Ernst F, Grizzle A. Drug related morbidity and mortality: Updating the cost-of-illness model. J. Am. Pharm. Assoc. 2001; 41(2):192-9.

18. Lazarou J, Pomeranz BH, Coreys PN. Incidence of Adverse Drug Reactions in Hospitalized Patients. JAMA. 1998; 279:1200-5.

19. Guillén DG, Albarracín A, Entralgo PL, Arquiola E, Montiel L, Peset JL, et al. História do Medicamento. Rio de Janeiro: Glaxo do Brasil S. A.; 1993.

20. American Society Health-System Pharmacists. ASHP guidelines on pharmacist-conducted patient education and counseling. Am. J. Health-Syst. Pharm. 1997; 54:431-4.

21. Cipolle R, Strand L, Morley P eds. Pharmaceutical care practice. New York: Mcgraw-HILL; 1998. p. 121-75: The patient care process.

22. Soares MA org. Medicamentos não prescritos: aconselhamento farmacêutico. 2.ed. Lisboa: Publicações Farmácia Portuguesa; 2002. p. 23-64: Automedicação e o farmacêutico.

23. Soares MA org. Medicamentos não prescritos: aconselhamento farmacêutico. 2.ed. Lisboa: Publicações Farmácia Portuguesa; 2002. p. 65-114: Cuidados farmacêuticos: Consulta, informação e comunicação com o paciente.

24. BRASIL. Congresso Nacional. Lei no 5.991, de 17 de dezembro de 1973. Dispõe sobre o controle sanitário do comércio de drogas, medicamentos, insumos farmacêuticos e correlatos, e da outras providências. Diário Oficial da [República Federativa do Brasil]. Brasília, 19 dez. 1973. [capturado 20 fev. 2007]. Disponível em: http:/ / e-legis.anvisa.gov.br/leisref/public/showAct.php?id=16614\&word=

25. Marques FB. Erros de Medicação. Informação Terapêutica. 2000; 4:3-5.

26. Sebastião ECO. Avaliação do Cumprimento das Exigências Legais em Ordens Médicas em Serviço de Farmácia Hospitalar de Ouro Preto e Implicações na Qualidade Assistencial ao Paciente. Rev. Ciênc. Farm. 2002; 23(1):71-85.

27. Cohen MR. Error-prone unit-dose packages concentration confusion apply the JCAHO "two-identifier" rule in the pharmacy. Hospital Pharmacy. 2004;39(8):726-8.

28. Ministério da Saúde. Agência Nacional de Vigilância Sanitária. Medicamentos. Glossário de Definições Legais [homepage na Internet]. Brasília: Ministério da Saúde; [capturado 20 fev. 2007]. Disponível em: http://www.anvisa.gov.br/medicamentos/glossario/glossario_m.htm 
29. BRASIL. Agência Nacional de Vigilância Sanitária. Resolução RDC nº. 135, de 29 de maio de 2003. Aprova regulamento técnico para medicamentos genéricos. Diário Oficial da [República @Ref numero texto = Federativa do Brasil]. Brasília, 2 jun. 2003. [capturado 20 fev. 2007]. Disponível em: http://e-legis.anvisa.gov.br/leisref/public/showAct.php?id=7909\&word=\#'

World Health Organization [homepage na Internet]. Ge-

30. neva: World Health Organization [capturado 22 out. 2004]. Disponível em: http:/ / www.who.int.

Maxwell S, Walley T. Teaching safe and effective prescri-

31. bing in UK medical schools: A core curriculum for tomorrow's doctors. Br. J. Clin. Pharmacol. 2003; 55:496-503.

USC School of Pharmacy [homepage on the Internet]. Los

32. Angeles: University of Southern California; c1905-2007 [capturado 13 jun. 2007]. Disponível em: http://www.usc.edu/schools/pharmacy/pharmd/.

Nontraditional Doctor of Pharmacy Program [homepage

33. on the Internet]. Dubai: Purdue University, School of Pharmacy and Pharmacal Sciences; c1869-2007 [atualizada em 12 maio 2005; capturado 13 jun. 2007]. Disponível em: http:/ / www.phpr.purdue.edu/ ntdpp/index.htm.

34. BRASIL. Congresso Nacional. Lei no 6.360, de 23 de setembro de 1976. Dispõe sobre a vigilância sanitária a que ficam sujeitos os medicamentos, as drogas, os insumos farmacêuticos e correlatos, cosméticos, saneantes e outros produtos, e dá outras providências. Diário Oficial da [República Federativa do Brasil]. Brasília, 24 set. 1976. [capturado 20 fev 2007]. Disponível em: http: / /e-legis.anvisa.gov.br/leisref/public/showAct.php?id=16615\&word=

35. Osório-de-Castro CGS, Castilho SR. Diagnóstico da Farmácia Hospitalar no Brasil. Rio de Janeiro: ENSP; 2004.

36. Hanlon JT, Weinberger M, Samsa GP, Schmader KE, Uttech KM, Lewis IK, et al. A Randomized, Controlled Trial of a Clinical Pharmacist Intervention to Improve Inappropriate Prescribing in Elderly Outpatients With Polypharmacy. American Journal of Medicine. 1996; 100(4):428-37.

37. Shelton PS, Fritsch MA, Scott MA. Assessing Medication Appropriateness in the Elderly: A Review of Available Measures. Drugs Aging. 2000; 16(6):437-50.
38. Bates D, Gawade A. Improving safety with information technology. N. Engl. J. Med. 2003; 348 (25):2526-34.

39. IHI.org [homepage on the Internet]. Cambridge: Institute of Health Improvement; c1991-2007 [capturado 25 out. 2004]. Disponível em: http:/ /www.ihi.org/.

40. Institute for Safe Medication Practices. List of error-prone abbreviations, symbols and dose designations. ISMP Medication Safety Alert [periódico na Internet]. 2003 [acesso em 22 Out 2004]; 8 (24):3-4 [aproximadamente 2 p.] Disponível em: http://www.ismp.org/msarticles/whitepaper.html

41. Food and Drug Administration. Medication errors. FDA Medical Bulletin. 1997; 27(2):1-3.

42. Leape LL, Brennan TA, Lard N, Lawthers AG, Localio AR, Barnes BA, et al. The nature of adverse events in hospitalized patients. Results of the Harvard Medical Practice Study II. N. Engl. J. Med. 1991; 324(6):377-84.

43. Holquist $C$, Phillips J. How FDA reviews drug names. Drug Topics. 2001; 36(1):1-3.

44. Noto A, Carlini E, Mastroianni P, Alves V, Galduróz J, Kuroiwa $W$ et al. Analysis of prescription and dispensation of psychotropic medications in two cities in the sate of São Paulo, Brazil. Rev. Bras. Psiquiatr. 2002; 24(2):68-73.

45. Barker K, Flynn E, Pepper G, Bates D, Mikeal R. Medication errors observed in 36 health care facilities. Arch. Intern. Med. 2002; 162:1897-903.

46. Lilley L; Guanci R. Look-alike abbreviations: prescriptions for confusion [Med Error]. Am. J. Nursing. 1997; 97(11):12.

47. Vieira RdCPA. Propagandas de medicamentos distribuídas aos médicos: aspectos legais, farmacológicos e de mercadização. Rio de Janeiro; 2004. Doutorado [Tese] - Universidade Estadual do Rio de Janeiro.

\section{CONFLITOS DE INTERESSE}

Declarou não haver

\section{ENDEREÇO PARA CORRESPONDÊNCIA}

Joice Mara Cruciol-Souza

Rua Rubens Carlos de Jesus, 111 - casa 28

Terras de Santana 2

86055-240 - Londrina - Paraná - Brasil

E-mail: jcruciol@uel.br ou jcruciol@yahoo.com.br 